\title{
A Study of Uterine Pathology in Patients Presenting With Abnormal Uterine Bleeding
}

\author{
Dr. P.M.Balaji ${ }^{1}$, Prof.B.Pushpa ${ }^{2}$, Dr.Subitha ${ }^{3}$, Dr.Sofiya. $C^{4}$ \\ ${ }^{1}$ Assistant Professor, Department Of Pathology, Govt. Kilpauk Medical College, Chennai \\ ${ }^{2}$ Professor, Department Of Pathology, Govt. Kilpauk Medical College, Chennai \\ ${ }^{3}$ Assistant Professor, Department Of Pathology, Madras Medical College, Chennai \\ ${ }^{4}$ Assistant Professor,Department Of Pathology, Govt. Kilpauk Medical College, Chennai
}

\begin{abstract}
:
Backgroud: Abnormal uterine bleeding (AUB) is the commonest presenting symptom in Gynaecology outpatient department. Endometrial sampling could be effectively used as the first diagnostic intervention in AUB, although at times, its interpretation could be quite challenging to the practicing pathologists. This study was done with hysterectomy specimen to evaluate histopathology for identifying the various etiologies in the age group between 45-60yrs presenting with $A U B$.

Objective: To determine the histopathologic spectrum of lesions associated with AUB in age group between 45 60yrs.

Material and Methods: This retrospective study was done at Government kilpauk Medical College,Chennai, India with hysterectomy specimen of 165 patients who presented with AUB from Jan2015-July 2016. Data including age and clinical indication for hysterectomy was collected for the study. The specimens were processed routinely and stained with hematoxylin and eosin stain and examined microscopically
\end{abstract}

Keywords: AUB, Histopathological lesions

\section{Introduction}

Endometrium is a dynamic, hormonally sensitive and responsive tissue which constantly and cyclically undergoes changes in the active reproductive life.Abnormal uterine bleeding is defined as a bleeding pattern that differs in frequency or duration or amount from a pattern observed during a normal menstrual cycle.It is one of the most frequently encountered and perplexing condition in women. AUB not associated with an organic cause, is referred as Dysfunctional Uterine Bleeding (DUB) [10]The AUB is one of the commonest complaints leading to endometrial sampling. Those ppatients with AUB who underwent hysterectomy were chosen.In this study, we have analysed the histopathological findings of various organic lesions in cases of AUB.

\section{Causes Of Abnormal Uterine Bleeding}

Abnormal uterine bleeding (AUB) may be due to structural abnormalities of the uterus. Some of the more common structural causes of abnormal uterine bleeding include benign (non-cancerous) lesions of the uterus such as polyps, fibroids (myomas), and adenomyosis (uterine thickening caused by endometrial tissue moving into the outer walls of the uterus) (Figure 1). Other causes include bleeding associated with early pregnancy, including miscarriage and ectopic pregnancy, as well as bleeding disorders that affect the ability of the blood to clot normally. Lesions of the cervix or vagina (benign and cancerous), chronic infections of the endometrial lining (endometritis), scar tissue (adhesions) in the endometrium, and the use of an intrauterine device (IUD) also may be associated with abnormal uterine bleeding.

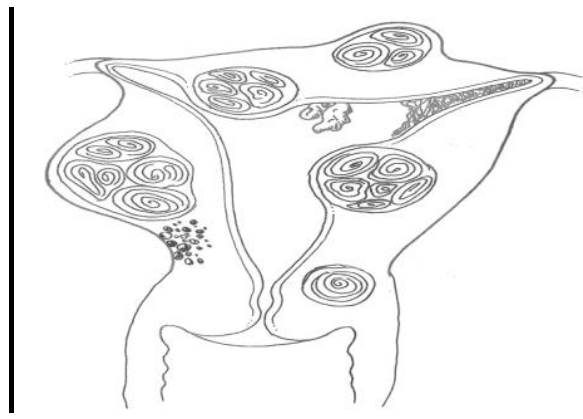

Estimates of the prevalence of adenomyosis vary widely from 5\% to 70\%[2] which is probably related to inconsistencies in the histopathologic criteria for diagnosis.On the contrary, leiomyomas have a high prevalence up to $70 \%$ in Caucasians and $80 \%$ in women of African ancestry[3,11]. They also have a wide 
spectrum of size and location (subendometrial, intramural, subserosal or a combination of these). Furthermore, they have widely varying rates of growth even in a single individual.

The purpose of our study was to find the prevalence of organic causes of AUB in the age group of 45-60yrs by histopathological examination of hysterectomy specimens received in our department.

\section{Materials And Methods}

A retrospective study was carried out in the pathology department from Jan 2015-july 2016. During this period, 350 hysterectomy specimens were received in the pathology laboratory in the hospital. Out of these, 165 were included in the study. Inclusion criteria were women coming to outpatient department with complaint of AUB for which hysterectomy was performed.Vaginal and abdominal hysterectomies done for complaints other than AUB and malignancies were excluded from the study.A record of these patients was retrieved and patient's age,parity, presenting symptoms, and clinical indication for hysterectomy was recorded. On gross examination, any apparent abnormality i.e., asymmetrical enlargement of the uterus, fibroid, polyp, any pinpoint/cystic areas of hemorrhage and endometrial thickening was noted. The number, size and location of the fibroid were also noted.A minimum of two sections were taken from the cervix,two from the corpus, one from both the tubes and ovaries and an additional section was taken from the leiomyoma or any other abnormal area. Representative sections were stained with hematoxylin eosin ( $\mathrm{H}$ and $\mathrm{E})$ stain and examined microscopically.

\section{Results}

A total of 165 cases were included in the study. Age of the patients ranged from 45 to 60 years. The largestgroup $(n=80)$ was of age $(45-50$ years) contributing $48.48 \%$ of total cases in the study [Table no-1].In this age group(45-60), leiomyoma was the most common pathology $52.12 \%(n=86)$ followed by adenomyosis $15.76 \%(n=26)$ where as $6.67 \%(n=11)$ showed dual pathology of adenomyosis and leiomyoma [Table 1]. In our study, leiomyoma was the most common histopathological lesion $(n=86)$ in women who presented with abnormal uterine bleeding [Table 1].

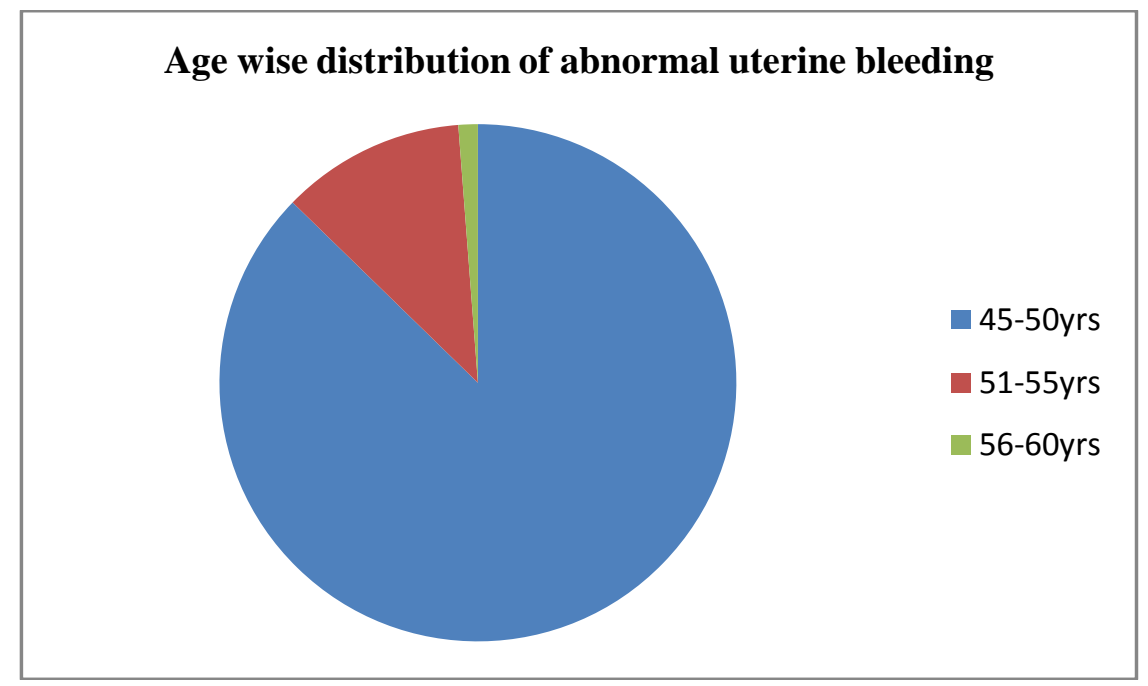

Table No:1

\begin{tabular}{|l|l|l|l|l|}
\hline Diagnosis & $\begin{array}{l}45-50 \mathrm{yrs} \\
\mathrm{No}(\%)\end{array}$ & $\begin{array}{l}51-55 \mathrm{yrs} \\
\mathrm{No}(\%)\end{array}$ & $\begin{array}{l}56-60 \mathrm{yrs} \\
\mathrm{No}(\%)\end{array}$ & $\begin{array}{l}\text { Total } \\
\mathrm{No}(\%)\end{array}$ \\
\hline Leiomyoma & $80(48.48)$ & $6(3.63)$ & 0 & $86(52.12)$ \\
\hline Adenomyosis & $23(13.93)$ & $3(1.81)$ & 0 & $26(15.76)$ \\
\hline Leiomyoma+Adenomyosis & $9(5.45)$ & $1(0.60)$ & $1(0.60)$ & $11(6.67)$ \\
\hline Endometrial Polyp & $1(0.60)$ & $3(1.81)$ & 0 & $4(2.42)$ \\
\hline Leiomyoma+Em Polyp & $3(1.81)$ & $1(0.60)$ & 0 & $4(2.42)$ \\
\hline Adenomyosis+Em Polyp & 0 & $1(0.60)$ & 0 & $1(0.60)$ \\
\hline Endometrial Hyperplasia & $5(3.03)$ & 0 & 0 & $5(3.03)$ \\
\hline Ein & $1(0.60)$ & 0 & 0 & $1(0.60)$ \\
\hline Endometrial Carcinoma & 0 & $1(0.60)$ & $2(1.21)$ & $3(1.81)$ \\
\hline Leiomyoma+Ovarian Cyst & $5(3.03)$ & $2(1.21)$ & 0 & $7(4.24)$ \\
\hline $\begin{array}{l}\text { Endometrial Polyp+Ovarian } \\
\text { Cyst }\end{array}$ & $2(1.21)$ & 0 & 0 & $2(1.21)$ \\
\hline Endocervical Polyp & $3(1.81)$ & 0 & 0 & $3(1.81)$ \\
\hline Cervical Carcinoma & $1(0.60)$ & 0 & $1(0.60)$ & $2(1.21)$ \\
\hline
\end{tabular}


A Study Of Uterine Pathology In Patients Presenting With Abnormal Uterine Bleeding

\begin{tabular}{|l|l|l|l|l|}
\hline Chronic Cervicitis & $1(0.60)$ & $1(0.60)$ & 0 & $1(1.21)$ \\
\hline Atrophic Endometrium & 0 & 0 & $5(3.03)$ & $5(3.03)$ \\
\hline Disordered Proliferative & $3(1.81)$ & 0 & 0 & $3(1.81)$ \\
\hline Total & $144(87.27)$ & $19(11.5)$ & $2(1.21)$ & $165(100)$ \\
\hline
\end{tabular}
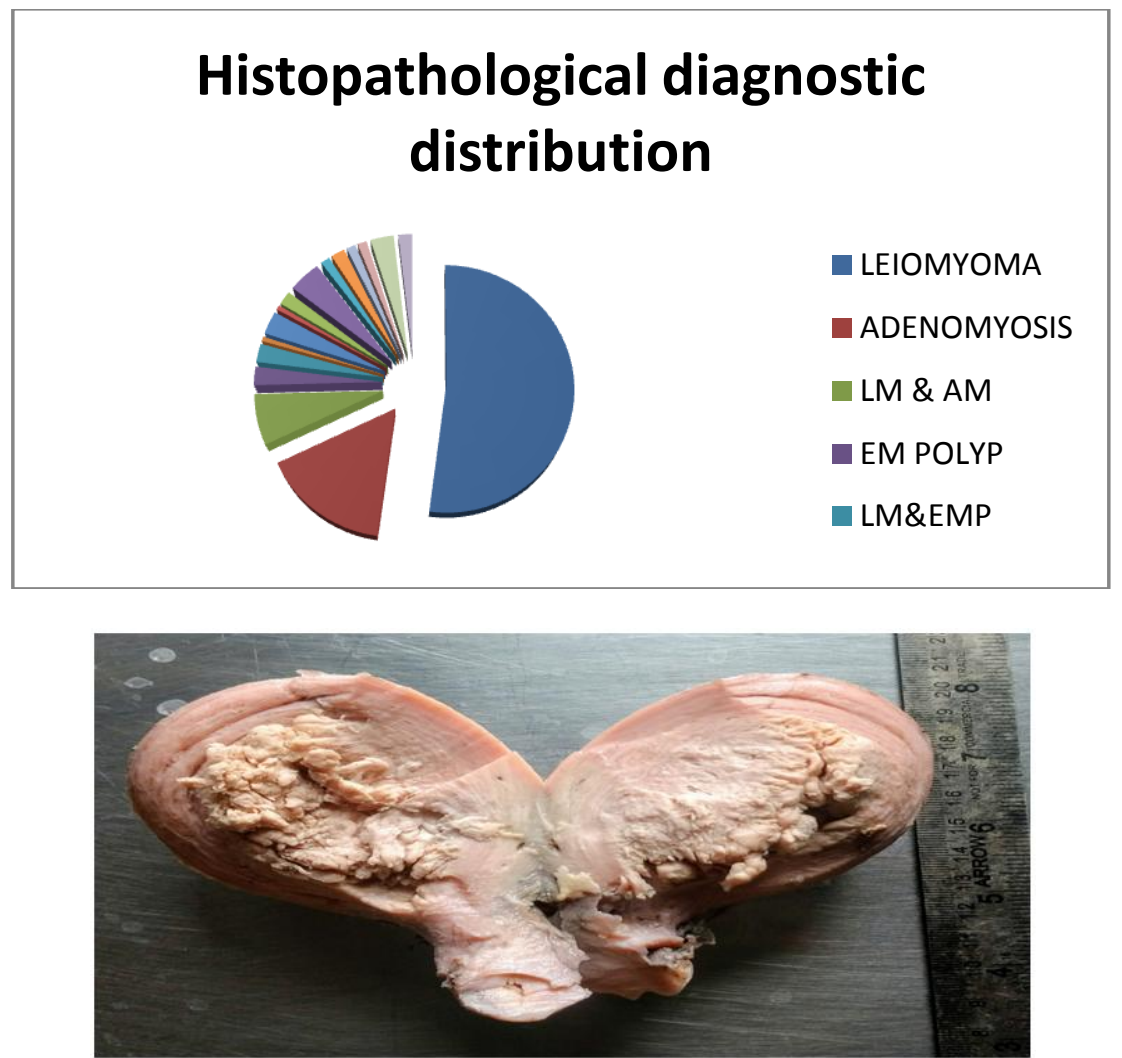

Fig 1: Gross picture showing growth in the Endometrium

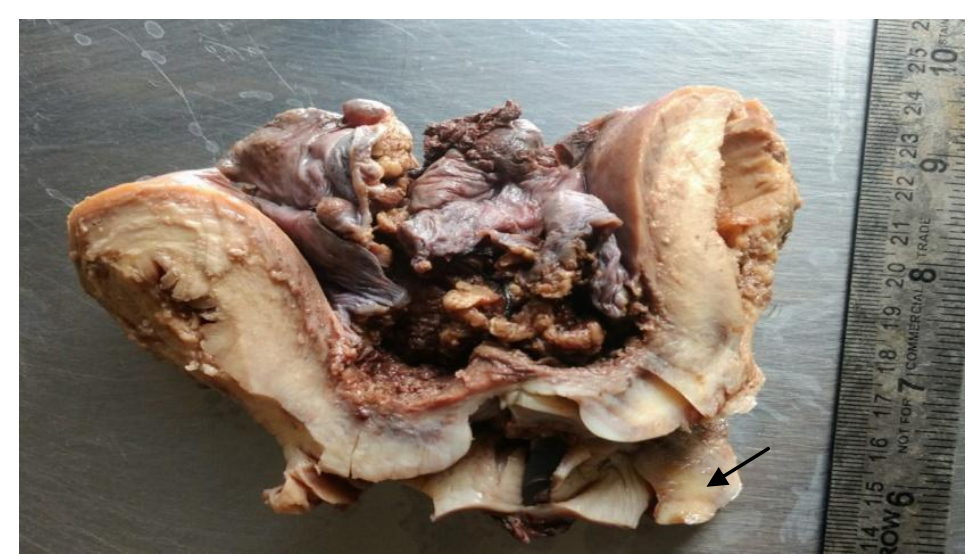

Fig 2: Gross picture showing growth in the cervix.

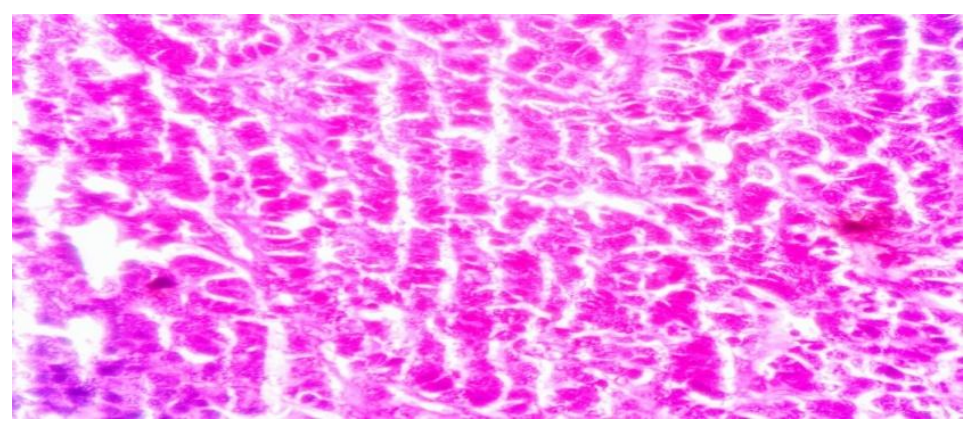

Fig 3 : Microscopic picture showing features of Nonkeratinising squamous cell carcin oma cervix. 100x 


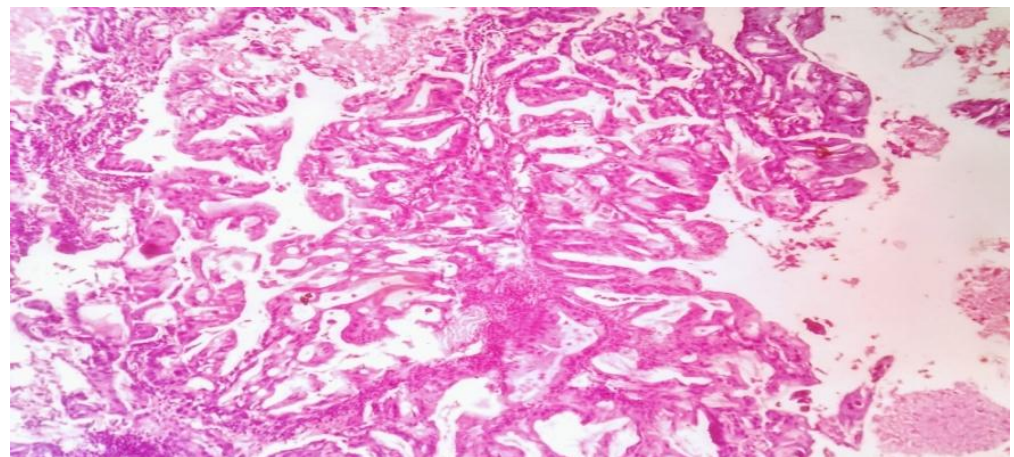

Fig 4 : Endometrial carcinoma- Type 2. 100x.

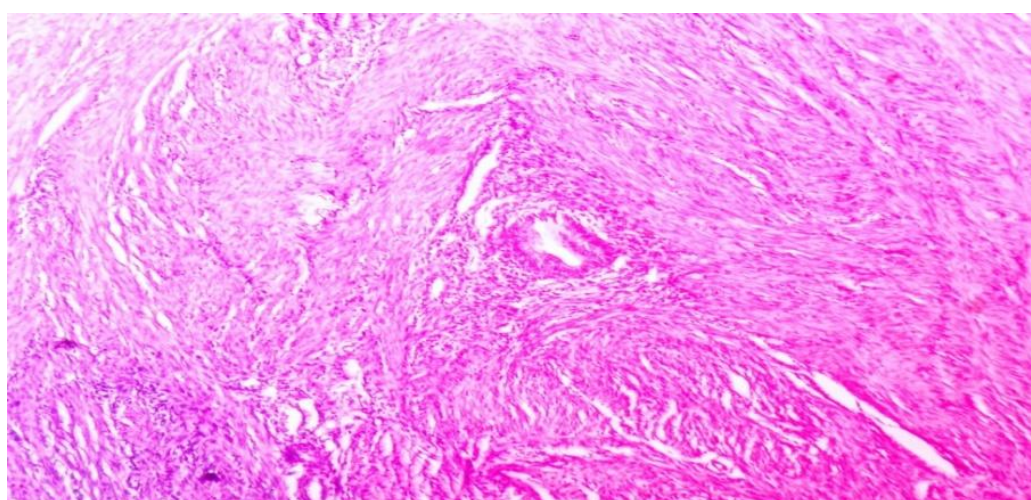

Fig 5 : Picture of Adenomyoma- Showing normal Endometrial gland and stroma surrounded by fascicles of benign spindle cells. 100x.

\section{Discussion}

The term abnormal uterine bleeding has been used to describe any bleeding not fulfilling the criteria of normal menstrual bleeding. The causes of abnormal uterine bleeding include a wide spectrum of diseases of the reproductive system and non-gynecologic causes as well.Organic cause of abnormal uterine bleeding maybe subdivided into reproductive tract disease, iatrogenic causes and systemic disease. When an organic cause of AUB cannot be found, then by exclusion, a diagnosis of dysfunctional uterine bleeding (DUB) is assumed. In about $25 \%$ of the patients, the abnormal uterine bleeding is the result of a well defined organic abnormality [9].

the occurrence of menstrual disorders increases with advancing age. The commonest age group presenting with excessive bleeding in our study was $45-50$ years. A similar incidence was reported by Yusuf et al. and Muzaffar et al. in their study of endometrium [1,2].

Our study like several others showed that proliferative lesions like disordered proliferative pattern, hyperplasia, and benign endometrial polyp occur more commonly in the age group 45-50 years [2]. The reason for increased incidence of abnormal uterine bleeding in this age group (45-50 years) may be due to the fact that these patients are in their climacteric period. As women approach menopause, cycles shorten and often become intermittently anovulatory due to a decline in the number of ovarian follicles and the estradiol level. The incidence of AUB between 51 and 60 years was lower as compared to those between 41 and 50 years. The

reason for this finding may be due to the fact that the patients were evaluated much earlier and treated appropriately thereby decreasing the incidence in later age group. We had only 2 patients with AUB in the age group of 55 to 60 years and one of them had endometrial carcinoma.

Predominant number of cases in this study showed normal physiologic phases such as proliferative, secretory and atrophic menstrual pattern. The bleeding in the proliferative phase may be due to anovulatory cycles[12] and bleeding in the secretory phase is due to ovulatory dysfunctional uterine bleeding.

A few number of cases showed disordered proliferative pattern in this study. Disordered proliferative pattern lies at one end of the spectrum of proliferative lesions of the endometrium that includes carcinoma at the other end with intervening stages of hyperplasias. The term "disordered proliferative endometrium" has been used in a number of ways and is somewhat difficult to define. It denotes an endometrial appearance that is hyperplastic but without an increase in endometrial volume [3]. It also refers to a proliferative phase endometrium that does not seem appropriate for any one time in the menstrual cycle,but is not abnormal enough to be considered hyperplastic.

Disordered proliferative pattern resembles a simple hyperplasia, but the process is focal rather than diffuse. A higher incidence of disordered proliferative pattern was found in our study as compared to Cho Nam- 
Hoon et al.[4]. An earlier stage of presentation due to increased health awareness could explain the high incidence in our study.Diagnosing the patients at the earliest stage of this spectrum will be of definite help to the practicing gynaecologists to prevent the disease progression. But pathologists should have clear cut criteria for diagnosing disordered proliferative pattern and this should not become a waste paper basket diagnosis. Atrophic endometrium was seen predominantly in the 55-60 years age group. The incidence is slightly lower when compared with results shown by Gredmark et al. [5].The exact cause of bleeding from the atrophic endometrium is not known. It is postulated to be due to anatomic vascular variations or local abnormal hemostatic mechanisms. Thin walled veins, superficial to the expanding cystic glands make the vessel vulnerable to injury.

The incidence of endometrial hyperplasias in this study was less as compared to others [5]. The possible explanation could be that most of patients here belong to lower socioeconomic status and the occurrence of risk factors like obesity, diabetes, increased intake of animal fat and sedentary life style is low. Another reason could be that most of these patients are being identified at a much earlier stage that is in the disordered proliferative phase. Identification of endometrial hyperplasia is important because they are thought to be precursors of endometrial carcinoma.

The incidence of benign endometrial polyps in this study was high in 45-55 years age group. Lower incidence of the endometrial polyps in the younger age group may be attributed to a possible spontaneous regression mechanism, which is characteristic of the cycling endometrium in reproductive age group. There is significant difference between the endometrial polyp and normal endometrium in receptor expression, cell proliferation and apoptosis regulation. These differences combined with non-random chromosomal aberrations and monoclonality suggests that polyp may provide a suitable microenvironment for the development of malignancy [6].

In the present study incidence of carcinoma endometrium was more common in the 51-60 years age group.The result of this study was almost similar to data mentioned by Yusuf et al. and Escoffery et al. in their study $[1,7]$. A study done by Dangal et al. in Nepal documented a lower incidence of endometrial cancer in Nepalese woman attributing it to the practice of early childbearing and multiparty [8]. Possibly, the same factors contributed to a lower incidence of carcinoma in our patients. Chronic endometritis was observed in few patients. One case showed epithelioid granulomas suggestive of tuberculosis. Patient with chronic endometritis can present with AUB, pelvic pain and infertility. This condition needs to be diagnosed because with specific treatment, endometrium starts functioning normally.

\section{Conclusion}

Uterine adenomyosis and leiomyoma are the most common benign conditions found in hysterectomy specimens with peak incidence at 45-55 years. Histopathology is mandatory for confirming diagnosis and ensuring optimal management.

\section{References}

[1]. Yusuf NW, Nadeem R, Yusuf AW, et al. Dysfunctional uterinebleeding. A retrospective clinicopathological study over 2 years.Pak J Obstet Gynaecol. 1996;9:27-30.

[2]. Muzaffar M, Akhtar KAK, Yasmin S, et al. Menstrual irregularitieswith excessive blood loss: a clinico-pathologic correlation.J Pak Med Assoc. 2005;55:486-9.

[3]. Steven SG. Problems in the differential diagnosis of endometrial hyperplasia and carcinoma. Mod Pathol. 2000;13:309-27.

[4]. Nam-Hoon C, Chan-II P, In-Joon C. Clinicopathologic study of the endometrium. Dysfunctional uterine bleeding. Korean J Pathol. 1989;23:65-74.

[5]. Gredmark T, Kvint S, Havel G, et al. Histopathological findings in women with postmenopausal bleeding. B J Obstet Gynaecol.1998;102:133-6.

[6]. Hileeto D, Fadare O, Martel M, et al. Age dependent association of endometrial polyps with increased risk of cancer involvement. World J Surg Oncol. 2005;3:8

[7]. Escoffery CT, Blake GO, Sargenat LA. Histopathological findings in women with postmenopausal bleeding in Jamaica. West Indian Med J. 2002;51:232-5.

[8]. Dangal G. A study of endometrium of patients with abnormal uterine bleeding at chitwan valley. Kathmandu Univ Med J.2003; $1: 110-2$.

[9]. Brenner PF. Differential diagnosis of AUB. Am J Obstet Gynecol.1996;175:766-9.

[10]. Albers JR, Hull SK, Wesley RM. Abnormal uterine bleeding. Am Fam Phys. 2004;69:1915-26.

[11]. Litta P, Merlin F, Saccardi C, et al. Role of hysteroscopy with endometrial biopsy to rule out endometrial cancer in post menopausal women with abnormal uterine bleeding. Maturitas. 2005; 50:117-23.

[12]. Kilbourn CL, Richards CS. Abnormal uterine bleeding, diagnostic considerations,Management options. Postgrad Med.2001; 109:137-50 